\title{
PERCEPCIÓN DE LA DISTINCIÓN ENTRE OCLUSIVAS SORDAS Y SONORAS INICIALES DEL INGLÉS (LE) POR ESTUDIANTES ARGENTINOS: DATOS DE IDENTIFICACIÓN Y DISCRIMINACIÓN
}

\author{
PERCEPTION OF THE DISTINCTION BETWEEN VOICELESS AND VOICED INITIAL \\ STOPS IN ENGLISH BY ARGENTINEAN LEARNERS: IDENTIFICATION AND \\ DISCRIMINATION DATA
}

\author{
Ubiratã Kickhöfel Alves \\ Universidade Federal do Rio Grande do Sul - Brasil \\ ukalves@gmail.com \\ Pedro Luis Luchini \\ Universidad Nacional de Mar del Plata - Argentina \\ luchinipedroluis@gmail.com
}

\begin{abstract}
En este trabajo, verificamos si el tiempo de inicio de voz (en inglés, Voice Onset Time - VOT) corresponde a la principal pista acústica utilizada en la identificación y discriminación de sonoridad de los segmentos oclusivos del inglés. Investigamos un grupo de 15 estudiantes de inglés, hablantes de español rioplatense como $\mathrm{L} 1$, los cuales realizaron un test de identificación y otro de discriminación $(\mathrm{AxB})$ en que se testearon cuatro patrones de VOT del inglés: (i) VOT Negativo y VOT Cero, que ocurren variablemente en las producciones de oclusivas sonoras; (ii) VOT Positivo, producido en oclusivas sordas y (iii) VOT Cero Artificial, un patrón manipulado y construido a partir del corte de aspiración de /p/, /t/, / / / . Los resultados confirman que el VOT no corresponde a la pista mayoritariamente seguida por los estudiantes, y que las distinciones de sonidos en la interlengua no son establecidas a partir de las mismas pistas acústicas que son funcionalmente primordiales entre hablantes nativos del inglés.
\end{abstract}

Palabras-clave: VOT; Percepción; Adquisición de LE; Español; Inglés

Keywords: VOT; Perception; FL Acquisition; Spanish; English

In this article, we investigate if Voice Onset Time (VOT) is the main acoustic cue employed by Argentinean students in the identification and discrimination of voicing in word-initial stops in English. Fifteen advanced learners of English participated in the study. Participants took an Identification and a Discrimination $(\mathrm{AxB})$ test, in which four VOT Patterns were investigated: (i) Negative VOT and Zero VOT, which occur variably in the production of voiced stops; (ii) Positive VOT, found in voiceless stops and (iii) Artificial Zero VOT, a manipulated pattern built with the extraction of aspiration in $/ \mathrm{p} /, / \mathrm{t} /, / \mathrm{k} /$. The results confirm that VOT is not the main acoustic cue followed by learners, and that voicing distinctions in the interlanguage are not based on the same acoustic cues employed by native speakers of English. 
(Recibido: 29/1/15; Aceptado: 17/9/15)

\section{Introducción}

En el idioma inglés, el Voice Onset Time $(V O T)^{1}$ es el principal correlato acústico responsable de la distinción entre las categorías fonológicas de "sordo" y "sonoro" en las oclusivas. Mientras que en inglés las oclusivas sonoras son sobretodo producidas con un VOT Cero (Lisker y Abramson 1964; Cho y Ladefoged 1999), y los segmentos sordos son producidos por medio de un VOT Positivo (voincing lag), tales patrones no son los mismos que los encontrados en idiomas como el francés, el español o el portugués brasileño. Conforme lo explican diversos autores, tanto el portugués (Istre 1983; Klein 1999) como el español (Lisker y Abramson 1964; Abramson y Lisker 1973; RAE 2011) presentan VOT Negativo (pre-voicing) en sus oclusivas sonoras y un patrón de VOT Cero en las consonantes sordas. En otras palabras, aunque el idioma español también presente una distinción funcional entre segmentos sordos y sonoros, esta distinción se produce fonéticamente de manera diferente a la encontrada en el idioma inglés.

En función de lo descripto, el reconocimiento de las diferencias entre segmentos oclusivos sordos y sonoros del inglés, por parte de estudiantes hispanohablantes y lusohablantes, implicaría un cambio en los patrones de VOT vigentes en el sistema de L1, si consideramos que el VOT corresponde a la pista acústica principal, utilizada por los estudiantes, en las distinciones entre oclusivas sordas y sonoras. No obstante, una serie de estudios que hemos desarrollado a lo largo de los últimos tres años, realizados con estudiantes brasileños del idioma inglés (Alves et al. 2011; Alves y Zimmer 2012, 2015), no nos han brindado evidencias sobre la importancia de esta pista entre hablantes brasileños. Datos obtenidos de aprendices de inglés, que tienen como sistema de L1 el dialecto portoalegrense del portugués brasileño, nos han demostrado que, aunque los estudiantes no suelen producir las oclusivas /p/, /t/, /k/ con la debida aspiración, son capaces de discriminar las oclusivas sordas de las sonoras en la lengua-meta.

Con base en estos resultados, en una serie de estudios, Alves et al. (2011) y Alves y Zimmer (2012, 2015) afirman que los altos índices de corrección en la identificación y discriminación de las oclusivas del inglés pueden deberse a que los estudiantes brasileños, a diferencia de los hablantes nativos del inglés americano (Schwartzhaupt et al. 2013), no toman el VOT como pista acústica principal en la distinción entre las oclusivas sordas y sonoras en el inglés. En otras palabras, es posible que pistas acústicas adicionales, además del VOT, estén siendo prioritariamente empleadas por los estudiantes en las distinciones de sonoridad. Algunos casos similares han sido discutidos en estudios tales como los de Sundara (2005), Oh (2011) y Kong et al. (2012). En esos trabajos -en los que se investigaron el francés canadiense, el coreano y el japonés como lengua materna- pistas acústicas adicionales (tales como la intensidad de la explosión (en inglés, burst) del segmento oclusivo y el valor de F0 (frecuencia fundamental) de la vocal siguiente) se mostraron determinantes para la distinción entre oclusivas sordas y sonoras. En estos idiomas, el VOT ejerce el rol de una pista adicional, que no es por sí misma suficiente para estimular distinciones perceptivas de sonoridad en los estudiantes. Dada esta posibilidad, la adquisición de las distinciones de sonoridad en inglés implicará que los estudiantes con estos sistemas de L1, en primer lugar, aprendan a focalizar su atención en la pista del VOT, para que puedan darse cuenta y aprender el nuevo patrón (aspiración) que ocurre en la lengua extranjera.

\footnotetext{
${ }^{1}$ En fonética, el tiempo de inicio de voz (en inglés, Voice-onset Time, VOT) es una característica de la producción de consonantes oclusivas. Se define como el intervalo de tiempo entre la explosión de la consonante oclusiva y el inicio de voceo de la siguiente vocal (Kent y Read 2002).
} 
La adquisición de la aspiración del inglés por estudiantes de esas lenguas, por lo tanto, corresponderá a una doble tarea: antes de aprender el patrón de VOT del inglés per se, los estudiantes tendrán que aprender a "escuchar" esta pista, que no tiene demasiada importancia en su L1.

La necesidad de aprender a escuchar esta pista se muestra clara cuando consideramos las consecuencias de lo que implica la falta de atención al VOT. Si los estudiantes de inglés no direccionan su atención al VOT, pero sí a otras pistas que también se muestran presentes en la señal acústica, es posible que, aun así, no tengan dificultades en identificar y discriminar $/ \mathrm{p} /, / \mathrm{t} / \mathrm{l} / \mathrm{k} / \mathrm{y} / \mathrm{b} /$, /d/, /g/, dado que estas otras pistas acústicas pueden también llevarlos a obtener altos índices en los procesos de discriminación e identificación en la lengua meta. Es importante aclarar que las oclusivas sordas /p/, /t/, /k/, por ejemplo, presentan mayor intensidad de explosión y valores más altos de $\mathrm{F} 0$ que /b/, /d/, /g/, también en idiomas en los que el VOT no es la pista mayoritariamente empleada en las distinciones de sonoridad. En función de lo anteriormente mencionado, el hecho de que los estudiantes no encuentren dificultad en discriminar dichas consonantes podría, a primera vista, llevarnos a concluir que no sería necesario concentrarse en el VOT. Sin embargo, si los estudiantes no centran su atención en esta pista, es posible que tampoco la utilicen en la producción de los sonidos del inglés y, como consecuencia, no produzcan la correspondiente aspiración ${ }^{2}$. Cabe resaltar que la falta de producción de aspiración por parte de los estudiantes puede tener efectos adversos en relación con la inteligibilidad del fenómeno (Jenkins 2000, 2007; Luchini 2004; Chiusano y Luchini 2010; Luchini y Kennedy 2013), en virtud de que los hablantes nativos de inglés utilizan la pista VOT (aspiración) para distinguir los segmentos sordos de los sonoros.

Considerándose que, así como en portugués, el español tampoco presenta aspiración en las oclusivas sordas, en este trabajo verificaremos si el VOT es la pista a la que mayoritariamente prestan atención estudiantes argentinos que tienen un dominio avanzado de inglés. Para alcanzar este objetivo, replicaremos los experimentos ya realizados con estudiantes brasileños (Alves et al. 2011; Schwartzhaupt et al. 2013; Alves y Zimmer 2012, 2015), con hablantes nativos de español bonaerense de la ciudad de Mar del Plata, provincia de Buenos Aires, Argentina. En estos experimentos, los alumnos brasileños completaron una batería de tests de identificación y discriminación de palabras del inglés iniciadas por segmentos oclusivos. Estas palabras eran iniciadas con el patrón de VOT Negativo (que puede ser encontrado variablemente en las producciones de /b/, /d/, /g/ iniciales del inglés, cfr. Lisker y Abramson 1964; Cho y Ladefoged 1999; Simon 2010) y VOT Positivo (que ocurre en /p/, /t/, /k/ iniciales en inglés, cfr. los autores ya mencionados). Se utilizaron, además, otros dos patrones de VOT como estímulo: uno natural y otro manipulado. El primero de ellos, que corresponde a un estímulo natural, se constituye por palabras del inglés iniciadas con $/ \mathrm{b} /, / \mathrm{d} /, / \mathrm{g} /$, exhibiendo el patrón VOT Cero, que puede ser encontrado variablemente en las producciones de las oclusivas sonoras del inglés (Lisker y Abramson 1964; Docherty 1992; Simon 2010), así como en las producciones de /p/, /t/, /k/ en español (Lisker y Abramson 1964; Abramson y Lisker 1973; RAE 2011) y en portugués (Istre 1983; Klein 1999). El segundo patrón acústico fue manipulado a partir de palabras del inglés con /p/, /t/ y /k/ iniciales. Para la elaboración de este patrón, se cortó completamente la aspiración de estas consonantes, de modo que este nuevo estímulo presentara el patrón de VOT de una consonante sonora del inglés (patrón VOT Cero), al mismo tiempo en que preservara todas las demás pistas acústicas (tales como intensidad de la explosión y F0) encontradas en una oclusiva inicial aspirada del inglés.

\footnotetext{
${ }^{2}$ Es necesario destacar que el presente trabajo no tiene como objetivo principal analizar los efectos e implicancias de las prácticas de instrucción explicita o de entrenamiento perceptivo para la adquisición del VOT. Sin embargo, destacamos la importancia y los beneficios producidos por la instrucción explicita para el logro de este objetivo (ver Alves y Magro 2011).
} 
Estos dos diferentes tipos de VOT Cero (natural y manipulado) podrán indicarnos si el VOT es la principal pista acústica considerada por estudiantes argentinos al identificar y discriminar la sonoridad de las oclusivas iniciales en inglés. La identificación del patrón VOT Cero Natural (/b/, /d/, /g/ del inglés) como caracterizador de un segmento sordo puede indicar que los estudiantes están siguiendo el patrón de VOT de su lengua materna, una vez que, en español, las consonantes /p/, /t/ y / / / exhiben este patrón. Por otra parte, la identificación de estas consonantes como sonoras puede llevarnos a considerar dos posibilidades diferentes: (i) los estudiantes ya toman el VOT como pista acústica principal en la distinción de sonoridad del inglés, y también ya han aprendido que la ausencia de aspiración corresponde a las oclusivas /b/, /d/, /g/ en este idioma; (ii) el VOT no es la principal pista acústica seguida por los estudiantes; en este caso, ni el patrón de VOT de la L1, ni tampoco el de la LE, está siendo seguido por los alumnos. Si consideramos esta última posibilidad, podemos suponer que los participantes están percibiendo mayoritariamente otras pistas acústicas, que los llevarían a identificar las consonantes con VOT Cero Natural como sonoras.

Respecto al VOT Cero Manipulado, su identificación como sordo puede indicar que: (i) los estudiantes están siguiendo, mayoritariamente, el patrón de VOT de su L1, dado que las oclusivas sordas son producidas con VOT Cero en español. En este caso, se espera que el patrón VOT Cero Natural sea también identificado como sordo, una vez que ambos patrones (Cero Natural y Manipulado) son iguales, en términos de duración de VOT; (ii) los estudiantes están siguiendo otras pistas acústicas, tal como la intensidad de la explosión y el F0, una vez que las consonantes resultante de la manipulación, con excepción del VOT, mantienen todas las otras propiedades acústicas que caracterizan las oclusivas sordas de la lengua-meta. Teniéndose en cuenta esta posibilidad, las respuestas a los patrones Cero Natural y Cero Manipulado deberán ser diferentes. Por otra parte, una posible identificación del Cero Manipulado como sonoro puede indicar que los estudiantes ya utilizan el VOT como pista acústica mayoritaria, y además ya han aprendido los patrones de VOT del inglés, donde la ausencia de aspiración caracteriza las consonantes oclusivas sonoras.

Cabe mencionar nuevamente que el método experimental empleado con los estudiantes argentinos que participaron en este trabajo, y cuyos resultados se presentan aquí, es similar al que se empleó en investigaciones anteriores con estudiantes brasileiros (Alves et al. 2011; Schwartzhaupt et al. 2014; Alves y Zimmer 2012, 2015) y con hablantes nativos de inglés americano (Schwartzhaupt et al. 2013). En los experimentos con estudiantes brasileños, mientras el patrón VOT Cero Natural fue predominantemente identificado como sonoro, el Cero Manipulado fue asociado a consonantes sordas, indicando que el VOT no es la pista prioritariamente seguida por estos estudiantes. Por otra parte, los dados con hablantes americanos, residentes en el estado de Pennsylvania, mostraron que el VOT constituye una pista fundamental para las distinciones entre oclusivas sordas y sonoras en este idioma, dado que tanto el VOT Cero Natural como el Manipulado fueron identificados como representantes de oclusivas sonoras.

Considerándose las premisas antes mencionadas, partimos de la hipótesis de que los estudiantes argentinos presentarán resultados semejantes a los encontrados en los datos provenientes de los participantes brasileños, dado que existe una similitud entre estas dos lenguas, en particular con respecto a los patrones de sonoridad de las oclusivas. Con base en esta motivación inicial, en este trabajo proponemos los siguientes objetivos generales: (i) investigar si estudiantes argentinos de inglés, con un nivel avanzado de la lengua, son capaces de distinguir la producción de diferentes patrones de VOT en las consonantes del inglés; (ii) discutir el estatus del VOT como una pista suficiente para las distinciones de sonoridad en inglés (LE) por parte de estudiantes brasileños y argentinos. 


\section{Método}

\subsection{Participantes}

Participaron en el experimento 15 estudiantes argentinos, que cursaban la carrera de Traductor Público en Inglés en una institución de enseñanza superior de la ciudad de Mar del Plata (Buenos Aires, Argentina). Todos los participantes nacieron y han vivido toda su vida en esta ciudad o en ciudades aledañas. Para determinar el dominio de los estudiantes en la lengua inglesa, todos los participantes rindieron el Oxford Online Placement Test ${ }^{3}$. Los resultados de este test indicaron que todos los participantes presentaban un nivel avanzado: niveles $\mathrm{C} 1$ o $\mathrm{C} 2$, conforme el Marco Común Europeo. Todos los participantes realizaron un Test de Identificación y un Test de Discriminación de sonidos en inglés.

\subsection{Diseño del Experimento}

\subsubsection{Estímulo de audio}

El estímulo fue grabado en un estudio profesional por 6 hablantes nativos de inglés (3 hombres y 3 mujeres), que estaban residiendo en Brasil por un período no mayor a 6 meses. Los seis locutores leyeron un conjunto de tres pares mínimos del inglés (bit-pit; dick-tick; gill-kill), cada par iniciado por una oclusiva con un diferente punto de articulación y seguido de una vocal frontal alta (Yavaș y Wildermuth 2006; Yavaș 2008). Para que pudiéramos garantizar la cualidad del estímulo, se solicitó que cada locutor leyera su lista de palabras tres veces consecutivas, lo que posibilitó la elección de los mejores estímulos perceptivos (en inglés, Tokens) para la elaboración del experimento.

Las oclusivas producidas por los 6 locutores presentaban tres patrones diferentes de VOT. Las oclusivas sordas (pit, tick, kill) fueron siempre pronunciadas con el patrón de VOT Positivo, mientras que /b, d, g/ (bit, dick, gill) fueron, en algunos casos, realizadas en forma alternativa, con VOT Negativo y, en otros, con VOT Cero, dado que los patrones de VOT en /b, d, g/ iniciales son variables en inglés (Lisker y Abramson 1964; Docherty 1992; Simon 2010).

Además de estos tres patrones, las oclusivas sordas iniciales fueron manipuladas por medio del programa Praat - Version 5.3.82 (Boersma y Weenink 2014) para la obtención del patrón Cero Manipulado. Al cortar el VOT de las oclusivas aspiradas, obtuvimos un segmento que presentaba el mismo valor de VOT de un segmento sonoro, pero con todas las otras características acústicas de una oclusiva sorda del inglés.

Ambos tests de Identificación y Discriminación fueron elaborados con los cuatro patrones de VOT: VOT Negativo, VOT Positivo, Cero Natural y Cero Manipulado. Los tests fueron elaborados y administrados por medio del programa Praat. En las secciones a continuación, se describen los testes empleados.

\subsubsection{El Test de Identificación}

El Test de Identificación consistía en una tarea en la cual los estudiantes debían reconocer en la pantalla de una computadora la consonante inicial de la palabra escuchada $(/ \mathrm{p} /, / \mathrm{b} /, / \mathrm{t} /, / \mathrm{d} /, / \mathrm{k} /$, $/ \mathrm{g} /)$.

\footnotetext{
${ }^{3}$ https://www.oxfordenglishtesting.com. Para más informaciones sobre el examen, véase Purpura (2007) y Pollitt (2007).
} 
El test presentaba 48 cuestiones en las que se incluyó cada uno de los 4 patrones de VOT descritos en la sección anterior (VOT Negativo, VOT Positivo, VOT Cero Natural y VOT Cero Manipulado). Cada uno de esos patrones contó con 12 estímulos perceptivos (4 para cada punto de articulación). La tarea fue realizada en el laboratorio de la universidad.

\subsubsection{El Test de Discriminación}

El Test de Discriminación era una tarea AXB. Los participantes escuchaban secuencias de tres palabras y debían responder si la consonante inicial de la segunda palabra era la misma que la de la primera palabra (e.g. bit - bit - pit), que la de la tercera palabra (p. ej. bit - pit - pit), o si las tres palabras tenían la misma consonante inicial (p. ej. pit - pit - pit).

Tres tipos de contrastes fueron testeados en la tarea AXB: VOT Negativo vs. VOT Positivo, VOT Negativo vs. Cero Artificial y VOT Cero Artificial vs. Positivo. El test presentaba 12 cuestiones para cada contraste, con 4 cuestiones para cada punto de articulación ${ }^{4}$, totalizando 36 cuestiones. Además de los tres tipos de contrastes verificados en el experimento, también fueron presentadas 9 cuestiones (tres para cada punto de articulación) en el formato "catch trial" - esto es, secuencias de tres palabras que presentaban el mismo patrón de VOT inicial (p. ej. pit - pit- pit), con el objetivo de verificar si los participantes estaban realmente prestando atención a la tarea ${ }^{5}$. El test fue realizado en el laboratorio de la universidad.

\section{Hipótesis}

\subsection{Test de Identificación}

En la elaboración de las hipótesis, prevemos que, de la misma manera que ocurre con aprendices brasileños de inglés (Alves et al. 2011; Schwartzhaupt et al. 2014; Alves y Zimmer 2012, 2015), el VOT no será la pista mayoritariamente seguida por los estudiantes argentinos. A continuación, presentamos las cuatro Hipótesis del Test de Identificación, referentes a cada uno de los patrones de VOT testeados:

Hipótesis 1: Los índices de identificación del VOT Negativo (producciones de /b/, /d/, /g/ en inglés) como "sonoro" serán significativamente más altos que los de "sordo" o "error"6.

De acuerdo con esta hipótesis, respecto a las oclusivas sonoras del inglés con VOT Negativo, hay una convergencia de pistas acústicas que llevan el oyente a identificar este segmento como un segmento sonoro. De hecho, las consonantes con VOT Negativo presentan el patrón de sonoridad del español, además de presentar una intensidad de la explosión débil, que también caracteriza los segmentos sonoros.

\footnotetext{
${ }^{4}$ Siguiendo a Alves y Zimmer (2013, 2015), otros contrastes posibles, tales como Cero Natural vs. VOT Negativo, bien como Cero Natural vs. Cero Artificial, no fueron incluidos. De hecho, Alves et al. (2011) han mostrado que, de estos dos contrastes, apenas el segundo suele ser discriminado por estudiantes que no tienen el VOT como pista acústica mayoritariamente responsable por las distinciones de sonoridad en su L1.

${ }^{5}$ Dado que las respuestas de los catch trials presentaron altos niveles de corrección (lo que indica, por lo tanto, que los participantes realmente prestaban atención a la tarea), los resultados de estas cuestiones no serán presentados en este artículo.

${ }^{6}$ Los "errores" corresponden a las respuestas en que los participantes optaron por un segmento con punto de articulación diferente de aquél que habían escuchado.
} 
En otras palabras, independientemente de la pista acústica mayoritariamente seguida por los estudiantes (el patrón de VOT de la lengua materna, de la lengua-meta u otras pistas acústicas, como la intensidad de la explosión), el resultado será el "sonoro".

Resultados similares fueron encontrados con estudiantes brasileños (Alves et al. 2011; Alves y Zimmer 2012, 2015; Schwartzhaupt et al. 2014), que no tienen el VOT como pista mayoritaria, bien como con hablantes del inglés americano (Schwartzhaupt et al. 2013), que toman esta pista como fundamental para las distinciones de sonoridad.

Hipótesis 2: Los índices de identificación del VOT Positivo (aspiración de /p/, /t/, /k/ en inglés) como "sordo" serán significativamente más altos que los de "sonoro" o "error".

Respecto a las oclusivas sordas aspiradas del inglés, hay una convergencia de pistas acústicas que llevan el oyente a optar por un segmento sordo. En términos de VOT, estas consonantes son consideradas como sordas tanto por los participantes que ya han adquirido el patrón de la lengua meta, como también por aquellos participantes que siguen el patrón de sonoridad de la L1 (una vez que estas consonantes no presentan vibración de las cuerdas vocales antes de la explosión). Respecto a la intensidad de la explosión, en el caso de ser esa la pista acústica mayoritariamente seguida por los estudiantes, tales consonantes presentan una explosión fuerte, lo que también los llevaría a optar por un segmento sordo. Resultados similares fueron encontrados en estudios realizados con estudiantes brasileños (Alves et al. 2011; Alves y Zimmer 2012, 2015; Schwartzhaupt et al. 2014), que no emplean el VOT como pista mayoritaria, a diferencia de los hablantes del inglés americano (Schwartzhaupt et al. 2013), que sí toman esta pista como fundamental para las distinciones de sonoridad.

Hipótesis 3: Los índices de identificación del VOT Cero sin manipulación (/b/, /d/, /g/ en inglés) como "sonoro" serán significativamente más altos que los de "sordo" y "error".

El patrón VOT Cero sin manipulación, por su vez, no presenta una convergencia entre las pistas acústicas de VOT e intensidad de la explosión. De hecho, si los participantes siguen el patrón de sonoridad de su L1, deberán identificar las consonantes como "sordas". Para que el segmento sea considerado sonoro, el estudiante (i) ya debe estar siguiendo, mayoritariamente, los patrones de VOT de la lengua meta; (ii) debe tomar otra pista acústica (tal como la intensidad de la explosión). La hipótesis 3 se elaboró a partir de esta segunda posibilidad, tomando como referencia los resultados obtenidos con los estudiantes brasileños (Alves et al. 2011; Alves y Zimmer 2012, 2015; Schwartzhaupt et al. 2014).

Hipótesis 4: Los índices de identificación del VOT Cero manipulado como "sordo" serán significativamente más altos que los de "sonoro" y "error".

Este patrón manipulado tampoco presenta una convergencia entre las pistas acústicas de VOT e intensidad de la explosión, siendo diferente del anterior por presentar todas las pistas acústicas que caracterizan las oclusivas sordas del inglés, con excepción del VOT. Por este motivo, la observación de las respuestas brindadas a los dos patrones de VOT Cero (Natural y Manipulado) puede revelar si el VOT corresponde a la pista acústica mayoritariamente seguida por los estudiantes. De acuerdo con la Hipótesis 4, consideramos que, al contrario de lo que ocurre con los hablantes nativos de inglés (Schwartzhaupt et al. 2013), y de modo similar a lo verificado con los estudiantes brasileños (Alves et al. 2011; Schwartzhaupt et al. 2014; Alves y Zimmer 2012, 2015), los participantes no considerarán el VOT como pista acústica mayoritaria, lo que resultará en la identificación de una consonante sorda. 


\subsection{Test de Discriminación}

Las hipótesis para este test también fueron elaboradas a partir de la idea inicial de que el VOT no es la pista acústica mayoritariamente seguida por los estudiantes. A continuación, presentamos las tres hipótesis del Test de Discriminación, cada una referente a un diferente tipo de contraste testeado.

Hipótesis 5: En las cuestiones de discriminación entre el VOT Negativo y el VOT Positivo, los índices de acuidad (discriminación) serán significativamente más altos que los de "igualdad" y de "error".

De acuerdo con lo anteriormente mencionado, tanto el patrón VOT Negativo como el VOT Positivo presentan una convergencia de pistas acústicas que llevan a los oyentes a considerarlos como sonoro y sordo, respectivamente. Por este motivo, se esperan altos índices de discriminación, conforme ya verificado en trabajos previos con estudiantes brasileños (Alves et al. 2011; Schwartzhaupt et al. 2014; Alves y Zimmer 2012, 2015), y con los propios hablantes nativos de inglés (Schwartzhaupt et al. 2013).

Hipótesis 6: En las cuestiones de discriminación entre el VOT Negativo y el VOT Cero Manipulado, los índices de acuidad (discriminación) serán significativamente más altos que los de "igualdad" y de "error".

En conformidad con esta hipótesis, los participantes de este estudio considerarán, como diferentes, los segmentos que presentan el mismo patrón de VOT, dado que las demás pistas acústicas del segmento manipulado corresponden a las de una consonante sorda.

Este resultado es diferente a lo verificado en la información proveniente de estudios realizados con hablantes nativos de inglés (Schwartzhaupt et al. 2013), que tienden a no discriminar estos dos patrones, porque toman el VOT como pista acústica fundamental para las distinciones entre "sordo" y "sonoro".

Hipótesis 7: En las cuestiones de discriminación entre el VOT Cero Manipulado y el VOT Positivo, los índices de igualdad serán significativamente más altos que los de "acuidad” y de "error".

A diferencia de lo verificado en estudios realizados con hablantes nativos del inglés (Schwartzhaupt et al. 2013), los estudiantes argentinos, al seguir prioritariamente otras pistas acústicas distintas del VOT, considerarán los dos patrones como representantes de consonantes sordas.

\section{Resultados y Discusión}

\subsection{Test de Identificación}

En la Tabla 1, se presentan los resultados del Test de Identificación

\footnotetext{
7 "Igualdad" corresponde a las respuestas en las que los participantes afirman que las tres palabras de la secuencia presentan la misma sonoridad. Por su vez, "error" corresponde a las respuestas en las que los estudiantes elijen equivocadamente los dos miembros de la secuencia con sonoridad igual (e.g., piensan que X es igual a A, cuando, en verdad, debería ser igual a B, y vice-versa).
} 


\begin{tabular}{c|ccc}
\hline Patrón & Sordo & sonoro & error \\
\hline \multirow{2}{*}{ VOT Negativo } & $\begin{array}{c}\mathbf{1 . 1 1 \%} \\
(2 / 180)\end{array}$ & $\begin{array}{c}\mathbf{9 7 . 7 8 \%} \\
(176 / 180)\end{array}$ & $\begin{array}{c}\mathbf{1 . 1 1 \%} \\
(2 / 180)\end{array}$ \\
VOT Cero & $\begin{array}{c}\mathbf{1 7 . 2 2 \%} \\
(31 / 180)\end{array}$ & $\begin{array}{c}\mathbf{7 7 . 7 8 \%} \\
(140 / 180)\end{array}$ & $\begin{array}{c}\mathbf{5 . 0 \%} \\
(9 / 180)\end{array}$ \\
VOT Cero Artificial & $\begin{array}{c}\mathbf{6 2 . 7 8 \%} \\
(113 / 180)\end{array}$ & $\begin{array}{c}\mathbf{3 3 . 8 9 \%} \\
(61 / 180)\end{array}$ & $\begin{array}{c}\mathbf{3 . 3 3 \%}(6 / 180) \\
\text { VOT Positivo }\end{array}$ \\
$\begin{array}{c}\mathbf{9 6 . 1 1 \%} \\
(173 / 180)\end{array}$ & $\begin{array}{c}\mathbf{2 . 7 8 \%} \\
(5 / 180)\end{array}$ & $\begin{array}{c}\mathbf{1 . 1 1 \%} \\
(2 / 180)\end{array}$ \\
\hline
\end{tabular}

Tabla 1 - Resultados del Test de Identificación

Según habíamos previsto, el patrón VOT Negativo es identificado como sonoro de manera prácticamente categórica. También habíamos supuesto que el patrón VOT Negativo es mayoritariamente identificado como sordo. Estos resultados no son sorprendentes, visto que, conforme aclarado en nuestras hipótesis, ambos patrones presentan una convergencia de pistas acústicas que llevan a los oyentes a identificarlos como sonoro y sordo, respectivamente. Como ya lo hemos anunciado previamente, estos resultados son esperables, independientemente de que la pista mayoritariamente tomada para las distinciones de sonoridad pueda ser el VOT.

Respecto a los hallazgos provenientes del patrón VOT Negativo, los tests de Friedman indicaron una diferencia significativa $\left(\mathrm{X}^{2}(2)=28.723, \mathrm{p}=.00\right)$ en la comparación entre respuestas para "sordo", "sonoro" o respuestas "erradas" (equívocos en los puntos de articulación). Los tests post-hoc de Wilcoxon, con corrección de Bonferroni, indicaron una diferencia significativa en la comparación entre las respuestas "sonoro" y "sordo" $(Z=-3.623, \mathrm{p}=0.000)$ y entre "sonoro" y "error" $(Z=-3.623, \mathrm{p}=0.002)$. Se confirmó, por lo tanto, la primera hipótesis. En relación con los resultados referentes al patrón VOT Positivo, los tests de Friedman también indicaron una diferencia significativa $\left(\mathrm{X}^{2}(2)=19.419, \mathrm{p}=.000\right)$ en la comparación entre las respuestas para "sordo", "sonoro" o las respuestas erradas. Los tests post-hoc de Wilcoxon, con corrección de Bonferroni, indicaron una diferencia significativa en la comparación entre las respuestas "sordo" y "sonoro" $(Z=-3.529, \mathrm{p}=.000)$ y entre "sordo" y "error" $(Z=-3.529, \mathrm{p}=.000)$. Se confirmó, también, la segunda hipótesis.

El patrón VOT Cero es, también, preferencialmente identificado como sonoro (77,78\%), aunque no con índices tan altos como aquellos encontrados en el VOT Negativo, en el que la elección de consonantes sonoras es casi categórica. Los tests de Friedman indicaron una diferencia significativa $\left(\mathrm{X}^{2}(2)=26.069, \mathrm{p}=.000\right)$ en la comparación entre los tres tipos de respuestas, y los tests post-hocs de Wilcoxon, con corrección de Bonferroni, indicaron una diferencia significativa entre los índices de respuesta para "sonoro" y "sordo" $(Z=-3.419, \mathrm{p}=.001)$, y para "sonoro" y error" $(Z=-3.420, \mathrm{p}=.001)$. Estos datos confirman la tercera hipótesis. Conforme a lo ya explicado anteriormente, la preferencia por la identificación del segmento como sonoro puede ser indicadora de dos posibilidades: (i) los participantes ya han adquirido el patrón de VOT de la lengua meta; (ii) los participantes no siguen el VOT, sino otras pistas acústicas (tal como la intensidad de la explosión, que los llevaría a identificar al estímulo como sonoro) en las distinciones de sonoridad. Los resultados que a continuación se brindan serán indicadores de que la segunda posibilidad es la más probable. 
Finalmente, el patrón Cero Manipulado fue preferencialmente identificado como sordo $(62,78 \%)$, aunque los índices nos indiquen que los participantes no se mostraban tan seguros en la identificación de la sonoridad de este elemento híbrido (visto que en el 33,89\% de las respuestas, la opción "sorda" fue la elegida). También en este caso, los tests de Friedman indicaron una diferencia significativa $\left(\mathrm{X}^{2}(2)=24.571, \mathrm{p}=.000\right)$ en la comparación entre los tres tipos de respuestas, y los tests post-hocs de Wilcoxon, con corrección de Bonferroni, indicaron una diferencia significativa entre los índices de respuesta para "sordo" y "sonoro" $(Z=-2.641, \mathrm{p}=.008)$, y para "sordo" y "error" $(Z=-3.424, \mathrm{p}=.001)$. Estos hallazgos confirman la cuarta hipótesis.

Los resultados obtenidos de los datos relacionados con los dos patrones de VOT Cero (Natural y Manipulado), que fueron considerados comparativamente, brindan evidencias adicionales a favor de la posibilidad de que el VOT no corresponde a la pista acústica mayoritariamente empleada por los estudiantes argentinos. El hecho de que el Cero Natural (/b/, /d/ y /g/ en inglés) fue significativamente identificado como sonoro nos permite considerar la posibilidad de que estos participantes, con nivel avanzado de dominio del inglés, no estén siguiendo el patrón de VOT de la L1 en sus distinciones de sonoridad. Asimismo, los datos referentes al patrón Cero Manipulado indican que los participantes no identifican la sonoridad en función de la presencia de aspiración. En otras palabras, ni el patrón de VOT de la L1, ni tampoco el de la LE, sirve como el criterio principal para las distinciones entre $/ \mathrm{p} / / \mathrm{t} / / \mathrm{k} / \mathrm{y} / \mathrm{b} / / \mathrm{d} / / \mathrm{g} /$ del inglés. Tomando en cuenta lo ya mencionado, es probable que los estudiantes estén empleando otra(s) pista(s) acústica(s) para diferenciar sonoridad. ¿Sería eso, a caso, el resultado de otra pista acústica mayoritaria, o de un conjunto de otras pistas que, unidas, sean capaces de representar las distinciones de sonoridad? Para dar respuesta a estas cuestiones, es necesario realizar nuevas investigaciones que tengan como objetivo principal determinar cuál(es) es(son) la(s) pista(s) que realmente se encuentran activas entre los estudiantes. Cabe aclarar que ya contamos con nuevas hipótesis en las que planteamos, entre otras cuestiones, investigar la intensidad de la explosión. Asumimos que una explosión fuerte (como la encontrada en el patrón manipulado) puede llevar los participantes a identificar las consonantes como sonoras, mientras que una explosión débil (como la del VOT Cero Natural) puede llevar a los participantes a identificar las consonantes como sonoras.

\subsection{Test de Discriminación}

En la Tabla 2, se presentan los resultados obtenidos del Test de Discriminación.

\begin{tabular}{c|ccc}
\hline Contraste & Acuidad & Igualdad & Error \\
\hline VOT Negativo & & & $\mathbf{1 1 . 1 1 \%}$ \\
vs. & $\mathbf{6 3 . 3 3 \%}$ & $\mathbf{2 5 . 5 6 \%}$ \\
$(114 / 180)$ & & \\
VOT Cero Artificial & & & $\mathbf{2 . 7 8 \%}$ \\
VOT Negativo & & $\mathbf{2 . 2 2 \%}$ & $(5 / 180)$ \\
vs. & $\mathbf{9 5 \%}$ & $(4 / 180)$ & \\
VOT Positivo & $(171 / 180)$ & & $\mathbf{1 1 . 6 6 \%}$ \\
VOT Cero Artificial & & $\mathbf{4 5 . 5 6 \%}$ & $(21 / 180)$ \\
vs. & & $(82 / 180)$ & \\
\hline VOT Positivo & $\mathbf{4 2 . 7 8 \%}$ & \\
\hline
\end{tabular}

Tabla 2 - Resultados el Test de Discriminación AxB Discriminación 
Conforme a lo previsto, los patrones VOT Negativo y VOT Positivo son fácilmente discriminados, de forma tal que el índice de acuidad sea casi categórico (95\%). De hecho, ambos patrones exhiben una convergencia de pistas acústicas, de modo que tanto el VOT cuanto las demás pistas acústicas (entre ellas, la intensidad de la explosión) llevan al participante a diferenciar los dos patrones, independientemente de la pista mayoritariamente seguida por los participantes. Los tests de Friedman indicaron una diferencia significativa $\left(\mathrm{X}^{2}(2)=26.471, \mathrm{p}=.000\right)$ en la comparación entre las respuestas correctas, incorrectas o sin discriminación, y los tests post-hocs de Wilcoxon, con corrección de Bonferroni, indicaron una diferencia significativa entre los índices de respuesta para "correcta" e "igual" $(Z=-3.475, \mathrm{p}=.001)$, y para "correcta" y "error" $(Z=-3.471, \mathrm{p}=.001)$. Se confirmó, por lo tanto, la quinta hipótesis.

Respecto a la discriminación entre VOT Negativo y VOT Cero Manipulado, los datos en la Tabla 2 nos muestran que los participantes también tienden a discriminar entre estos dos patrones, aunque no lo hacen categóricamente $(63,33 \%)$. Estos resultados son diferentes a los encontrados en los estudios realizados por Schwartzhaupt et al. (2013), en los que hablantes nativos de inglés, al tomar el VOT como pista mayoritaria para las distinciones de sonoridad, no discriminan entre estos dos patrones. Los tests de Friedman indicaron una diferencia significativa $\left(X^{2}(2)=21.655, p=.000\right)$ en la comparación entre respuestas correctas, incorrectas o sin discriminación, y los tests post-hocs de Wilcoxon, con corrección de Bonferroni, mostraron una diferencia significativa entre los índices de respuesta para "correcta" e "igual" $(Z=-2.872, \mathrm{p}=.004)$, y para "correcta" y "error" $(Z=-3.415$, $\mathrm{p}=.001)$. Estos datos confirman la sexta hipótesis. Los datos reunidos aquí indican que el patrón de VOT del inglés todavía no ha sido adquirido por estos alumnos con dominio avanzado del idioma, sino que (i) están tomando el patrón de la L1 como referencia, bien (ii) toman otras pistas acústicas, tal como la intensidad de la explosión, que operan conjuntamente. Teniendo en cuenta los resultados anteriormente discutidos respecto del Test de Identificación y los índices de respuesta referentes al próximo contraste a ser examinado, estamos en condiciones de defender la segunda posibilidad.

Finalmente, verificamos los resultados referentes al contraste entre VOT Cero Manipulado y VOT Positivo. A diferencia de los estudiantes americanos (Schwartzhaupt et al. 2013), los argentinos muestran cierta incongruencia en la discriminación de estos patrones como representativos de dos consonantes diferentes o de un mismo segmento (con 42,78\% de discriminación). Esta diferencia se muestra en términos de porcentaje de índices de "igualdad" sobre "discriminación". La dificultad se reflejó en el resultado del Test post-hoc de Wilcoxon, con corrección de Bonferroni, que no indicó una diferencia significativa entre los índices de acuidad e igualdad $(Z=-.507, \mathrm{p}=.612)$. Por lo tanto, no se confirmó la séptima y última hipótesis.

Estos datos parecen sugerir que, a diferencia de lo verificado con hablantes nativos, el VOT, aunque importante, no sea la pista acústica mayoritariamente utilizada por los estudiantes argentinos en las distinciones entre oclusivas sordas y sonoras del inglés. Si el VOT fuera la pista mayoritariamente seguida, los resultados referentes a "discriminación" serían significativamente superiores. Por otra parte, si la intensidad de la explosión hubiera sido tomada como la única pista empleada por los estudiantes, los índices de igualdad habrían sido estadísticamente superiores (conforme habíamos expresado en esta última hipótesis). De hecho, no se encontraron diferencias significativas entre las respuestas de "Discriminación" e "Igualdad"; por lo tanto, los resultados provenientes de este último contraste indican que las distinciones de sonoridad pueden estar siendo establecidas a partir de múltiples pistas acústicas que operan conjuntamente. La adquisición de la lengua meta implicaría, en verdad, la necesidad de focalizar estas distinciones de sonoridad provistas por la pista del VOT. 


\section{Conclusión}

En este artículo, discutimos el estatus del VOT como pista suficiente, entre estudiantes argentinos, con dominio avanzado del inglés como lengua extranjera, para las distinciones entre las consonantes inglesas $/ \mathrm{p} /, / \mathrm{t} /, / \mathrm{k} / \mathrm{y} / \mathrm{b} /, / \mathrm{d} /, / \mathrm{g} /$. Los resultados sugieren que el VOT no resulta ser la pista acústica mayoritariamente seguida por estos alumnos en las distinciones de sonoridad de las oclusivas. Consideramos la posibilidad de que existan otras pistas, actuando conjuntamente, que asuman el rol prioritario en estas distinciones de sonoridad.

Oportunamente, hicimos referencia a una serie de estudios previos, desarrollados con estudiantes brasileños, en los que se plantea que el VOT tampoco resulta ser la pista acústica mayoritaria utilizada por estos estudiantes para determinar las distinciones de sonoridad en el sistema de interlengua. Sin embargo, estos hallazgos no se condicen con otros provenientes de estudios similares llevados a cabo con participantes norteamericanos.

Si bien los datos de esta investigación nos han demostrado que el VOT no parece ser la pista principal reconocida por los estudiantes en las distinciones de sonoridad en la lengua meta, es importante destacar la necesidad de realizar futuras investigaciones con el objetivo de determinar cuál es la pista acústica principal, o si se puede verificar una acción conjunta de múltiples pistas empleadas por la mayoría de los estudiantes argentinos. Conforme ya lo hemos mencionado, estudios previos como los de Sundara (2005), Oh (2011) y Kong et al. (2012) sugieren que tanto la intensidad de la explosión (en inglés, bust) como la frecuencia F0 pueden tener un papel fundamental en estas decisiones. Sería interesante investigar, por lo tanto, si una de esas pistas corresponde a un criterio mayoritario en las distinciones de sonoridad, o si la distinción entre segmentos sordos y sonoros se establece a partir de un número de pistas acústicas que tienen acción conjunta.

Por un lado, la presente investigación tuvo como objetivo evaluar, por medio de una serie de cuatro patrones de VOT, los diferentes niveles de identificación y discriminación de sonoridad de los segmentos oclusivos del inglés. Esta etapa experimental se realizó con un grupo de estudiantes argentinos con un dominio avanzado del idioma. Por el otro, consideramos pertinente explorar el rol del VOT en diferentes etapas de adquisición del sistema de LE. Para lograr esto, será necesario contrastar, en un futuro, la percepción de estudiantes de inglés con diversos niveles de competencia lingüística: básico, intermedio y avanzado. Cabe cuestionar si el uso de múltiples pistas acústicas, conforme vemos en los datos del presente estudio, corresponde a una característica "heredada" del sistema de L1 de los estudiantes, o si es un proceso que tiene inicio durante el proprio proceso de adquisición de LE. Creemos, asimismo, que es necesario y fundamental determinar la(s) pista(s) a través de las cuales se instancian las distinciones de sonoridad en el sistema de L1 de estos participantes. En otras palabras, cabe averiguar si, en la L1, la distinción entre segmentos sordos/sonoros se da a través de la presencia o ausencia de VOT Negativo, o si otras pistas acústicas presentan, también, un rol fundamental en estas distinciones. A la fecha, estamos investigando estas cuestiones en relación con el dialecto del español empleado por hablantes monolingües del español, variedad bonaerense, nacidos en la ciudad de Mar del Plata. También, intentamos evaluar cómo estas cuestiones impactan a lo largo de las diferentes etapas de adquisición del inglés de los estudiantes, con el objetivo de determinar en qué momento de la trayectoria del aprendizaje las múltiples pistas acústicas entran en acción conjuntamente.

Los resultados de esta investigación brindan datos contundentes e importantes que pueden ser de gran utilidad para el desarrollo de investigaciones futuras en percepción y producción de LE. 
A su vez, pueden también ser considerados de gran validez para el avance del área de la enseñanza de lenguas extranjeras. Sabemos que existen otras pistas acústicas que pueden conducir a los alumnos a diferenciar entre oclusivas sordas y sonoras del inglés. Por consiguiente, no podemos dejar de considerar la posibilidad de que, en términos de producción, estos estudiantes también sean capaces de producir la distinción entre $/ \mathrm{p} /, / \mathrm{t} /, / \mathrm{k} / \mathrm{y} / \mathrm{b} /, / \mathrm{d} /, / \mathrm{g} /$ a través de otras pistas que no sea el VOT. El hecho de que el VOT presenta un carácter "redundante" en las distinciones de sonoridad de las oclusivas del inglés puede explicar los bajos valores de aspiración encontrados por los estudiantes que cuentan con sistemas de L1 que no presentan oclusivas aspiradas (Zimmer 2004; Alves 2007; Alves y Magro 2011; Veiga-França 2011; Schwartzhaupt 2012; Prestes 2013; Alves y Zimmer 2015). Como consecuencia de lo anteriormente expuesto, estos estudiantes pueden asumir que el VOT tampoco tiene importancia en la producción de los sonidos, lo que puede resultar en problemas de inteligibilidad.

Por lo tanto, consideramos de fundamental importancia la realización de futuras investigaciones que jerarquicen el papel de la instrucción explícita y del entrenamiento perceptivo, basados en el rol y la importancia de la aspiración en inglés. En este sentido, el presente estudio, al destacar que el VOT no corresponde a la pista acústica prioritaria para estudiantes argentinos, evidencia la gran complejidad del proceso de adquisición de LE. Los resultados obtenidos en esta investigación nos permiten verificar cuán relevante es para los estudiantes desarrollar la capacidad de identificar pistas acústicas que, aunque no sean del todo significativas en su L1, cumplen un rol vital entre los hablantes de la lengua meta.

\section{Referencias}

Abramson, Arthur S. y Leigh Lisker. 1973. Voice-timing perception in Spanish word-initial stops, Journal of Phonetics, 1: 1-8.

Alves, Ubiratã Kickhöfel. 2007. Uma discussão conexionista sobre a explicitação de aspectos fonético-fonológicos da LE: dados de percepção e produção da plosiva labial aspirada do inglês, em J. M. Poersch y A. A. Rossa (eds.), Processamento da Linguagem e Conexionismo, Santa Cruz do Sul, Editora da UNISC: 155-185.

Alves, Ubiratã Kickhöfel y Vivian Magro. 2011. Raising awareness of LE phonology: explicit instruction and the acquisition of aspirated /p/ by Brazilian Portuguese speakers, Letras de Hoje, 46(3): 71-80.

Alves, Ubiratã Kickhöfel y Márcia Cristina Zimmer. 2012. The dynamics of perception and production of VOT Patterns in English by Brazilian Learners, em E. Mello, M. Pettorino y T. Raso (eds.), Proceedings of the VIIth GSCP international conference: speech and corpora, Firenze, Firenze University Press: 223-227.

Alves, Ubiratã Kickhöfel y Márcia Cristina Zimmer. 2015. Percepção e produção dos padrões de VOT do inglês por aprendizes brasileiros: o papel de múltiplas pistas acústicas sob uma perspectiva dinâmica, Revista Alfa, 59(1): 157-180.

Alves, Ubiratã Kickhöfel, Bruno Moraes Schwartzhaupt y Ana Hemmons Baratz. 2011. Percepção e produção dos padrões de VOT do inglês (LE) por aprendizes brasileiros, en G. FerreiraGonçalves, M. R. Brum-de-Paula y M. Keske-Soares (eds.), Estudos em aquisição fonológica, Vol. 4, Pelotas, Editora e Gráfica Universitária da UFPel: 179-189.

Boersma, Paul y David Weenink. 2014. Praat - Doing phonetics by computer, Versión 5.3.82, Amsterdam [en línea]. Disponible en http://www.praat.org. 
Chiusano, Ana Christina y Pedro Luis Luchini. 2010. Implementing accuracy- and fluency-based tasks for the training of plosive consonants: a study conducted with Argentinean LE learners, COPAL - Concordia Working Papers in Applied Linguistics, 2: 56-77.

Cho, Taehong y Peter Ladefoged. 1999. Variation and universals in VOT: evidence from 18 languages, Journal of Phonetics, 27: 207-229.

Docherty, Gerald J. 1992. The timing of voicing in British English Obstruents, Berlin, Foris Publications.

Istre, Giles Lother. 1983. Fonologia transformacional e natural: uma introdução crítica, Florianópolis, Editora da UFSC.

Jenkins, Jennifer. 2000. The phonology of English as an international language, Oxford, Oxford University Press.

Jenkins, Jennifer. 2007. English as a Lingua Franca: attitude and identity, Oxford, Oxford University Press.

Kent, Ray e Charles Read. 2002. The acoustic analysis of speech. New York, Cengage Learning.

Klein, Simone. 1999. Estudo do VOT no Português Brasileiro, Tesis de Maestria, Universidade Federal de Santa Catarina, Florianópolis. Inédita.

Kong, Eun Jong, Mary E. Beckman y Jan Edwards. 2012. Voice onset time is necessary but not always sufficient to describe acquisition of voiced stops: the cases of Greek and Japanese, Journal of Phonetics, 40: 725-744.

Lisker, Leigh y Arthur S. Abramson. 1964. A cross-language study of voicing in initial stops: acoustical measurements, Word, 20:384-422.

Luchini, Pedro Luis. 2004. Developing oral skills by combining fluency- with accuracy-focused tasks: a case study in China, Asian EFL Journal, 1: 141-151.

Luchini, Pedro Luis y Sara Kennedy. 2013. Exploring sources of unintelligibility between nonnative English speakers: a case study, The International Journal of English and Literature, 4(3): 79-88.

Oh, Eunjin. 2011. Effects of speaker gender on Voice Onset Time in Korean stops, Journal of Phonetics, 39: 59-67.

Pollitt, Alastair. 2007. The meaning of OOPT Scores, Oxford [en línea]. Disponible en: https://www.oxfordenglishtesting.com

Prestes, Susana Pinheiro da Cruz. 2013. Produção de consoantes oclusivas iniciais do inglês por falantes do $P B$, Tesis de Maestria, Universidade Federal do Paraná, Curitiba. Inédita.

Purpura, James. 2007. The Oxford Placement Test: what does it measure and how?, Oxford [en línea]. Disponible en: http://www.oxfordenglishtesting.com

RAE - Real Academia Española. 2011. Nueva gramática de la lengua española - fonética y fonología, Barcelona, Espasa Libros.

Schwartzhaupt, Bruno. 2012. Factors influencing Voice Onset Time: analyzing Brazilian Portuguese, English and Interlanguage data, Trabajo Final de Carrera (Letras), Universidade Federal do Rio Grande do Sul, Porto Alegre. Inédita.

Schwartzhaupt, Bruno Moraes, Ubiratã Kickhöfel Alves y Ana Beatriz Areas da Luz Fontes. 2013. O VOT como pista suficiente para a distinção surdo/sonoro: dados de falantes do inglês americano. Trabajo presentado en el IV Seminário de Aquisição Fonológica, Santa Maria, Rio Grande do Sul, 10-13 septiembre 2013.

Schwartzhaupt, Bruno Moraes, Ana Hemmons Baratz y Ubiratã Kickhöfel Alves. 2014. Percepção e produção das plosivas do inglês (LE) por aprendizes brasileiros, Anais do I Seminário Internacional de Aquisição da Linguagem, Porto Alegre [en línea]. Disponible en: http://ebooks.pucrs.br/edipucrs/anais/sial/2011/src/31.pdf

Simon, Ellen. 2010. Voicing in contrast: acquiring a second language laryngeal system, Ghent, Academia Press.

Sundara, Megha. 2005. Acoustic phonetics of coronal stops: a cross-language study of Canadian English and Canadian French, Journal of the Acoustical Society of America, 118: 1026-1037. 
Veiga-França. Karoline. 2011. A aquisição da aspiração das plosivas surdas do inglês por falantes do Português Brasileiro: Implicações teóricas decorrentes de duas formas de descrição dos dados, Tesis de Maestria, Universidade Católica de Pelotas, Pelotas. Inédita.

Yavaş, Mehmet. 2008. Factors influencing the VOT of English long lag stops and interlanguage phonology, en A. S. Rauber, M. A. Watkins y B. O Baptista (eds.), New Sounds 2007: Proceedings of the fifth international symposium on the acquisition of second language speech, Florianópolis, UFSC: 492-498.

Yavaș, Mehmet y Renée Wildermuth. 2006. The effects of place of articulation and vowel height in the acquisition of English aspirated stops by Spanish speakers, IRAL, 44: 251-263.

Zimmer, Márcia Cristina. 2004. A transferência do conhecimento fonético-fonológico do português brasileiro (L1) para o inglês (LE) na recodificação leitora: uma abordagem conexionista. Tesis de Doctorado en Letras. Pontifícia Universidade Católica do Rio Grande do Sul, Porto Alegre. Inédita. 